\title{
Research on Multimodal Teaching Modal Reform for English Majors
}

\author{
Hong-yan Sun
}

School of Foreign Languages of Handan College

\begin{abstract}
This paper explores that in English major curriculum, the teachings of the courses for English majors should reform the classroom teaching mode through a variety of ways to establish an appropriate multimodal teaching mode. Teachers should employ the proper teaching modal according to the qualities of the courses in the choice of mode in the process of English Teaching and handle the synergy of the different teaching modals well. The different teaching modes of the courses cooperate to train the students' comprehensive quality and ability of knowledge. The multimodal teaching modal is one of the effective ways and means to improve the comprehensive quality of English majors.
\end{abstract}

Key words -English Major, Multimodal, Teaching Modal

\section{英语专业“多模态化”教学模式改革探究}

\author{
孙红艳 \\ 邯䣋学院外国语学院, 邯郸, 河北, 中国
}

\begin{abstract}
摘 要 本文重点探讨在英语专业课堂教学中, 英语专业课程应通过多种途径改革教学模式, 建立一个合适的多模态教学模式, 在英语教学的模态选择过程中, 教师根据课程特点在选择模态主导因素的基础上, 处理好不同模态之间的协同关系。各种课程不同模态 的教学模式相互协同,形成英语专业“多模态化”教学模式, 共同完成对学生知识和能力等综合素质的培养。英语专业“多模态化”教学模 式是有效地提高学生综合素质的途径和手段之一。
\end{abstract}

关键词 英语专业, 多模态化, 教学模式

1. 引言

随着高校教学改革的不断深入, 培养目标的不断提高, 和英语专业以学生为本的大类招生下的人才培养方案的需 求, 英语专业学生对教师的教学方式和方法要求和期望值 越来越高, 这要求教师转变教学理念, 引导学生在多种模态 下, 着重培养学生实际运用语言的能力, 以学生为中心, 充 分调动本专业学生主要学习英语语言和英美国家的社会、 文学、文化等方面的基础知识, 接受英语听、说、读、写、 译等方面的语言基本训练, 掌握中小学英语教学与管理等 方面的专业知识, 初步具备一定的业务水平、较高的文化 素质和教学工作能力。本文试图探讨在英语教育专业进行 多模态教学, 以学生为本, 突出质量意识, 以培养“毕业能 就业、就业能上岗、上岗能上手、上手成骨干、骨干留得 住”的应用型人才为根本任务, 加快推进英语专业大类招生 下的以学生为本的 “ $1+2+1$ ” 人才培养模式改革, 提高应用型
人才培养质量。

\section{2. 理论背景}

多模态话语研究兴起于 20 世纪 90 年代可以通过听觉、 视觉、触觉等多种反角, 通过语言、图像、声音、动作等 多种手段和符号资源进行交际的现象。顾曰国根据多模态 话语分析理论, 提出了多模态外语学习模型, 即用角色建 模语言来构建学习行为模型。学生通过模态所扮演的角色 来构建知识。该模型的主要内容是外部环境互动角色, 用 信息获取角色表示; 大脑对外部环境互动角色所获取的信 息进行处理, 用意义建构角色表示; 学习效果的外部行为 表现, 用实践能力角色表示。这种模态之间的互动, 以建 构学生的知识, 使学生的主体地位得以体现, 最终实现学 生的素质提高。随着多模态理论的完善和发展, 在英语教 育专业课堂教学中, 各门课程整合各种资源, 初步形成了 
不同的教学模式, 因此, 各种模态之间的协调合作显得尤 为重要, 为英语专业培养人才提供了一个良好的教学平台。

\section{3. 多模态课堂教学模式}

英语专业为了进一步搞好专业各门专业骨干课程的教 学方法方式的改革, 英语教育专业关于教学方法方式改革 的建设目标具体为改革骨干课程的教学方法和方式, 凸显 “多元化”教改特点, 逐步形成“多模态教学模式”的有特色 的专业课程教学方法方式。各课程模式应注重培养学生的 应用能力和创新精神, 完善现有教学模式, 并采用多种现 代化辅助教学手段, 将传统教学方法的精华与现代教育技 术相结合, 逐步提高英语专业的整体教学质量。

改革教学方式方法, 不同的课程采用不同的教学模式。 如在 “英美文学及选读”课程中实施 “四个三结合教学模 式”, “高级英语”课程中实施“前景化”教学模式, “综合英语” 课程中实施“TELOS”教学模式, “英文写作”课程中实施“双 过程化控制” 教学模式, “口译理论与实践”课程教学中实施 “3S”教学模式, “英语教学法”课程中实施“仿+练+研+评” 教学模式, 以及在实践教学中实施“ $4 \mathrm{C}$ ”能力人才培养模式 等等。

“四个三结合” 教学模式是在教学目标、教学内容、教 学手段和成绩评价四个方面的改革成果。“四个三结合”中 的第一个“三”是在教学过程中贯彻“双高+双强+双意识”三 结合的教学理念, 即把学生培养成思想素质高、专业水平 高、外语实践能力强、信息技术运用能力强、具有创新意 识和合作意识的复合型人才; 第二个“三”是实施“历史+文 选+评论” 三结合的教学模式, 即通过简述历史, 精选作品 篇章, 细评作品精华, 让学生学到知识, 运用好知识, 提 高学术的鉴赏水平和人文素养; 第三个 “三”是采用“提问 + 讲授+讨论” 三结合的教学手段, 即师生相互提问, 共同讲 授, 共同讨论, 锻炼学生的独立思考、正确表达自己思想 的潜在能力; 第四个 “三”是运用“课堂表现+课外作业+期末 测试”三结合的考核方法, 即学生的成绩由自主学习成果展 示、课堂讨论、话剧表演、论文写作、期末考试五个成绩 之和构成, 改变了传统的一张期末试卷定终身的终结性评 价, 注重对学生的学习过程性评价。“英美文学及选读”课 程“四个三结合”教学模式注重学生知识的拓展和综合能力 的培养, 通过“课前预习一课堂教学一自主学习一能力评 价”流程, 实现各项教学目标的完成。

“高级英语”课程“前景化”教学模式的构建, 为提高 学生的语言鉴赏力提供有效指引。第一, “前景化”教学模 式旨在实现教学内容的“前景化”, 即语言“前景化”是文学 语言最突出的特点之一, 教师可将文本中遵循常规语言表 达的内容作为学生自学或非重点教学内容, 而将偏离常规
语言表达的文本内容, 即“前景化”现象作为教学重点。例 如, 语音前景化、词汇前景化、语法前景化、语义前景化、 书写前景化、语域前景化、方言前景化、历史时期前景化。 采用根据内容讲解文本中的“前景化”现象, 达到突出重点 和解决难点的效果, 如运用语篇分析法从宏观上把握主旨 大意, 语法翻译法帮助学生理解难句; 文体分析法和语用 分析法挖掘原作深刻内涵, 提高欣赏能力。

第二, “前景化”教学模式旨在实现教学方法的“前景 化”; 即首先是作业前置、课下自主学习、课堂学生提问。 作业前置就是要求学生结合课后练习预习课文, 根据课后 练习发现文本中的重点内容, 而这些重点内容往往是偏离 常规表达的“前景化” 内容。课下自主学习就是将学生预习 时发现的“前景化”问题先查阅资料, 疑难问题在课堂上提 交小组讨论或提问教师解决。教师可对此部分问题进行精 讲, 同时对于学生自学解决的问题, 教师可让学生发表看 法和评论, 或选小组代表发言。教师授课和学生练习反馈 交错进行; 让学生自己写作, 大声回答, 表演出教材的片 段, 或对作家可能做出的选择加以猜测等等具体的教学方 法, 采用师生、生生互动的交际式、课堂小组讨论式相结 合的教学模式, 涵盖听、说、读、写、译五种技能的训练, 拓展学生文化视野, 培养综合分析能力, 同时积极提倡学 生自主学习。

第三, “前景化”教学模式旨在实现教学手段的“前景 化”, 本项目致力于借助多媒体技术手段辅助教学内容和方 法的“前景化”, 利用 PPT 的文本特征使“高级英语”教学内 容“前景化”。

“高级英语 “前景化”教学模式研究”的目标是运用多 种教学手段和策略引导学生参与课堂教学, 增加互动, 营 造生动活泼的氛围, 提高学生分析文章结构、主题、语言 特点、修辞手段及写作技巧的能力, 提高鉴赏能力、语言 运用能力和思辨能力, 同时注重培养学生的自主学习能力, 为日后深造打下基础。

英语专业实践教学“4C”人才培养模式的目标是完善实 践教学管理与成绩评价, 加强教学教材建设, 确定教师在 实践教学中的角色, 最终达到提高学生的英语语言与教师 职业技能所具备的交际能力 (communicating)、合作能力 (co-operating)、组织能力 (conducting) 和创新能力 (creating) 的目的, 是培养具有综合实力人才的有效途径。专业实践 教学的内容包括必修课程和选修课程。学生在指导教师的 指导下, 有计划地进行训练, 通过系列实践教学, 在语言 技能、语言知识、职业技能方面达到现代社会所需要的外 语人才的要求。主要实践课程为: 职业生涯设计、话剧表 演、读一本好书、词汇学习与测试、中西方文化知识学习 与测试、课件制作、模拟课堂、模拟谈判、模拟商务会谈、 
模拟领导人会见口译、教育见习、教育实习、参加技能和 知识大赛 (如朗诵、演讲、辩论、阅读、写作、翻译等)。

英语专业实践教学 “ $4 \mathrm{C}$ ” 人才培养模式” 吸取了国内 高校开放式实践教学成功的经验, 根据高校英语教育专业 人才培养目标, 制定了高校英语教育专业“开放式实践教学 “4C”人才培养模式, 为实现教育部提出的新时期外语人才 培养目标奠定了基础; 将若干实践教学和管理统一规划, 从不同的侧面培养学生的“ $4 \mathrm{C}$ ”能力, 使开放式的实践教学 更具整体性、系统性和可操作性, 使学生的成绩评定更有 科学性; 同时, 为教师教学角色改革提供了一条新的途径。 普通高校英语教育专业开放式实践教学 “ $4 \mathrm{C}$ ”人才培养模 式, 是针对英语教育专业学生存在的不足提出的, 它突出 准教师在英语教学中 “ $4 \mathrm{C}$ ”能力的培养, 为提高学生的教师 职业技能提出了一个新的培养模式。

综合英语 TELOS 教学模式是华东师范大学邹为诚教 授在开展综合英语课堂教学实践性探索的基础上 总结出 一个由“语言输入” (Target Language Input)、“学习投入” (Engagement)、“语言选择” (Learner's Active Choices)、 “语言输出” (Comprehensible Output) 和“错误处理”

(Selective Negative Input) 五要素组成的教学模式的简称。

TELOS 教学模式的实践效果表现在该模式对提高学 生的语言能力, 尤其是听说能力有效果显著。多层次、多 角度、多变化的目标语输入能激发学生的学习兴趣, 为提 高学生的听说能力奠定了基础; 精心设计的课堂教学、贴 近学生生活的课堂活动、有梯度、开放性的课堂问题是提 高学生听说能力的催化剂; 交际性的错误纠正既帮助学生 注意避免语言表达中的错误, 又保全了学生的面子, 是提 高听说能力的润滑液; 而有质量的语言输出是提高听说能 力的关键。

其次, TELOS 模式下的合作学习对提高学生的交际能 力有明显效果。通过合作学习, 学生的非认知能力 (性格; 自信心; 创新、合作精神; 社会交往能力) 均有一定的提 高, 它改善了学生的性格, 提高了学生的自信心, 增强了 学生的创新、合作精神和社会交往能力。著名的合作学习 理论专家郎和波特把合作学习的好处归纳为 5 条: 学习者 获得更多的语言使用机会; 学习者使用的目的语质量提高; 学习者的个体差异得到相当程度的照顾; 降低了学习者使 用陌生语言的焦虑感; 学习的积极性得到了提高。(Long $\&$ Porter,1985) 由此可以看出, 合作学习不只是一种学习的 方法, 还是一种生活的态度。

再次, TELOS 教学模式有利于延长学生学习的高峰 期, 推迟或延缓高原期的到来, 避免过渡语石化现象的产 生。TELOS 教学模式中的五要素不仅为教师因材施教、因 时制宜提供了各不相同的语言输入材料, 同时又照顾打学
生的情感因素, 避免学习焦虑感, 能充分调动学习英语的 热情和信心, 有利于延长学习高峰期。

再有, TELOS 教学模式有利于体现学习者个体差异, 培养学习积极性。个体差异是指个人在认知、情感、意志 等心理活动过程中表现出来的相对稳定而又不同于他人的 心理、生理特点。Altman 认为, 学习者个体差异因素主要 包括年龄、性别、过去的语言学习经验、母语熟练程度、 感觉方式偏爱、认知方式、学习策略等。在综合英语教学 中我们发现即使使用同样的教材, 在同样的教学环境中学 习, 学生的英语学习成绩有很大差别。i+1 输入模式能保证 向不同语言能力的学习者提供适合其语言水平的输入语; 教师通过课堂教学, 设计不同层次的课堂活动, 营造适合 学习者学习的“场”, 能满足不同认知方式学习者的需求; TELOS 教学模式对教师和教师课堂管理提出的具体要求, 以鼓励学生为主, 避免挫伤学习积极性, 为不同性格和不 同学习动机的学生营造了宽松的学习环境, 而课堂管理中 的“奖励”原则不仅有利于构建积极的学习态度, 还可提高 自主学习能力。

英文写作“双过程化控制写作模式”是由“启发过程” 和“写作过程”构成。“双过程”中的“启发过程”在写作课堂由 教师主持完成。教师通过讲解, 使学生了解写作技巧, 通 过实例演示, 激发学生的写作兴趣和鉴赏力, 通过组织课 堂讨论, 培养学生创新思维能力。九个步骤的“写作过程” 既是课堂教学向课外训练的延伸, 也是“启发过程”的继续。 为有效完成该过程, 教师应精心设计“启发过程”的每一个 环节，制定行之有效的训练计划。如“三位一体的训练计 划”, 即逻辑、语法、修辞的整体训练。教师通过讲解逻辑 思维技巧, 通过讲练如何正确地选词、写句、组织篇章结 构, 如何选用适当的修辞手段, 通过选用正反两个方面的 范文, 让学生讨论, 掌握语言的正确表达方法和策略, 避 免出现逻辑、语法、修辞等方面的错误。同时, “写作过程” 的九个步骤的有效实施, 也需要教师给予学生知识上的传 授和写作技能上的大量训练, 使学生对写作的每个步骤和 写作要求有一清晰的了解。学生只有在写作课上得到科学 有效的训练, 才能如期完成写作过程的九个步骤。总之, 教师通过实施“双过程化控制写作模式”, 运用“讲解+实例+ 讨论”三位一体的教学手段, 使学生掌握写作知识与技能, 为后续写作奠定思维、语言和技能基础。因此, 良好的课 堂训练是写作课“启发过程”的拓展, 是“写作过程”计划有 效实施的基础, 是学生体验课外快乐写作的可靠保障。

英语教学法采用“仿+练+研+评”教学模式, 课程内容选 择了教材中的五个单元, 包括英文板书和书写、英文歌曲 教唱、简笔画、教学微技能、语言知识与语言技能教学。 根据实际需要又增添了说评课、外语课堂活动设计两项内 
容。该项内容的选择将会帮助学生全面提升英语教学基本 功, 更新教学理念, 为今后教学工作打下坚实的基础。本课程 介绍了英文书写、英文歌曲、简笔画、课堂教学微技能、 语言知识与语言技能教学、外语课堂活动设计和实施等内 容; 旨在教会学生能够掌握一定的教学技能, 即通过案例 教学, 将优秀的实践方法移植到自己的教学实践中去; 通 过反思、讨论、探索、分析提高学的能力; 通过分析、评 价、讨论提高评价能力; 最终能够根据实际需要选择并运 用恰当的教学方法和技巧; 具备课堂管理的能力、驾驭教 材的能力; 分析问题和解决问题的能力, 为学生和教师进 行教学探索和教学方法与技巧的创新打基础。

英语口译人才培养“ $3 S$ ” 模式是在口译研究中的释意理 论、图示理论和人本主义教育理论的指导下, 主要由特定 的培养目标、教学内容和方法、课程体系以及管理制度和 评估方式构成, 四者缺一不可、互为补充。“3S”模式下成 功口译员的标准, 即英语口译人才培养“ “3S”模式下成功口 译员的标准: 即成功的口译=文化图式+口译技巧+口译准 则。

$3 \mathrm{~S}$ 模式下的人才培养, 旨在使学生通晓口译的本质、 基本理论和技巧, 了解口译记忆的规律和信息提取的过程, 掌握基本的口译方法和策略。3S 模式下口译人才培养的充 分考虑到课程体系设置的系统性、科学性和地方性, 解决 如何开设口译课程相关课程群的问题, 它实现了“两个结 合”, 即 “模块+层次”两个有机结合。模块分为 “基础知识教 育、口译专业知识教育、口译员职业方向教育和跨学科教 育有机地结合; 层次结构为基础课、专业课、跨学科课程 的有机结合。管理制度和评估方式: 本模式下的口译教学 努力引导学生自主地进行学习、提高口译学习的效率, 并 设置了开放的管理制度和评估方式。口译教学监督管理机 制是由学校、院系、辅导员、指导教师、小组组长和学生 个人六方面有机构成。同时实行日常训练的小组长负责制 和竞赛制。评估手段实行“5+3”, 即考勤、作业、课堂参与、 课堂测试、期末成绩五个方面相结合, 以及教师测评、合 作伙伴评价和实习单位评估三种形式相结合。

\section{4. 结论}

英语专业课程教学通过实施“多模态”教学模式, 教师 转变了教学理念, 以学生为中心, 引导学生在多种模态下, 培养实际运用语言的能力, 学生学习英语语言和英美国家 的社会、文学、文化等方面的基础知识兴趣得到提高, 在 英语听、说、读、写、译等方面的语言得到充分训练, 提 高了素质, 成为“毕业能就业、就业能上岗、上岗能上手、 上手成骨干、骨干留得住”的应用型人才, 为推进英语专 业 $1+2+1$ 人才培养模式的改革提供了可借鉴的依据, 为提 高英语专业应用型人才培养的质量奠定了坚实的基础。

\section{参考文献(Reference)}

[1] Yongsheng Zhu, The Basic Theory and Research Method of Multimodal Discourse Analysis. Foreign Language Journal, 2007, (05).

[2] Xuesong Chen, Research on Multi Modal Theory of University English Teaching Design. Journal of Changchun Finance College, 2012, (2).

[3] Yueguo Gu, Multimedia, Multimodal Learning Analysis. Computer-Assisted Foreign Education, Vol. 2, pp: 7-16, 2007.

[4] Delu Zhang, Research on Design of Modalities of Multimodal Foreign Language Teaching. Foreign Languages in China, 2010,(3)

[5] Zhang Delu and Wang Lu, "Synergy of Multimodal Discourse in Foreign Language Teaching," Foreign Language Research, 2010, (2).

[6] Delu Zhang, Research on the Multimodal Learning Ability Cultivation Model[J]. Foreign Language Research, 2010,(2).

[7] Xiaomei Wei, English Language Teaching Resourses Constructionin in the Perspective of Multimodal Discourse Theory. Hei Longjiang Education, 2012 (6). 